\title{
How the brain retains lymphoma cells
}

Both primary central nervous system lymphomas (CNSLs) and secondary CNSLs derived from peripheral sites can occur in the brain, but it is not clear how malignant lymphoma cells (LCs) invade and proliferate in the brain, especially as lymphoid cells are not typically present in this tissue. However, as chronic inflammation can promote development of peripheral lymphomas, and as the inflammatory nuclear factor- $\kappa \mathrm{B}(\mathrm{NF}-\kappa \mathrm{B})$ pathway is upregulated in CNSLs, O'Connor et al. hypothesized that chronic CNS inflammation might contribute to CNSL development.

The authors first chronically activated non-canonical NF- $\mathrm{kB}$ signalling in the CNS by creating transgenic mice that expressed the cytokines lymphotoxin $\alpha$ (LTa) and LT $\beta$ under the control of the astrocyte-specific glial fibrillary acidic protein (Gfap) promoter. Brains of these GFAP-LT $\alpha \beta$ mice had upregulated inflammatory pathways and widespread gliosis (a response to CNS injury), but no synaptic loss or disruption of the blood-brain barrier (BBB). Activation of chronic canonical NF- $\kappa \mathrm{B}$ signalling in astrocytes using inducible constitutively active I $\mathrm{B}$ kinase 2 (IKK2CA) also induced widespread gliosis without BBB disruption in mice.

Intravenous injection of GFAPLT $\alpha \beta$ mice or IKK2CA mice with $B$ cell LCs expressing the oncoprotein MYC (E $\mu-M Y C$ cells) led to more neurological disturbances and substantial LC colonization of the brain compared with wild-type mice. Similar results were observed with LCs expressing a different oncoprotein (TCL1).

To better understand the pathways by which LCs might enter and colonize the brain, the authors first investigated the role of various cell adhesion molecules (CAMs). Several endothelial cell (EC) CAMs were more highly expressed in
GFAP-LT $\alpha \beta$ brains than in wildtype brains, and the expression of these CAMs was increased in vitro and in vivo by activation of NF- $\kappa B$ signalling via $\mathrm{ACH} 6$, an agonist of the LT $\beta$ receptor. Furthermore, LC binding to ECs in vitro was also increased by ACH6. However, CAM upregulation was not sufficient to induce CNSL development.

Tracing of individual LCs in live mice using two-photon microscopy indicated that the number of LCs in the brain parenchyma was higher and that these cells were retained for longer in GFAP-LT $\alpha \beta$ mice compared with wild-type mice. LCs were capable of entering wild-type brains, but they rarely proliferated.

Other studies have indicated that the chemokine CXCL12 expressed in ECs limits immune cell retention in the brain, and indeed wild-type mice expressed CXCL12 in brain ECs. The authors hypothesized that LCs exit wild-type brains in response to this CXCL12, but that glial cells in GFAP-LT $\alpha \beta$ brains might produce a chemokine that counteracts CXCL12 to allow retention of LCs.

To identify this putative chemokine, the authors profiled chemokine expression in brains from GFAP-LT $\alpha \beta$ and IKK2CA mice. Several chemokines were elevated, but CCL19 was the only chemokine that remained high in brains from which the blood vessel-rich meninges had been removed, suggesting that it was produced in non-ECs. Furthermore, the CCL19 receptor CCR7 was expressed in both MYC- and TCL1-expressing LCs, indicating that CCL19-CCR7 signalling might mediate LC retention in the brain parenchyma.

Further experiments supported this hypothesis. First, CCL19 expression was higher in GFAP-LT $\alpha \beta$ primary astrocytes compared with wild-type, and activation of NF- $\kappa \mathrm{B}$ signalling in wild-type astrocytes increased CCL19 expression. Second, the authors showed that $\mathrm{Cr} 7^{-1-} \mathrm{LCs}$ could enter the brain parenchyma of GFAP-LT $\alpha \beta$ mice (albeit at a lower frequency than LCs expressing CCR7) but could not form CNSLs; similarly, CNSLs did not form in GFAP-LT $\alpha \beta$ mice that lacked CCL19 expression. Finally, in vitro migration assays indicated that CCL19 counteracted the ability of LCs to migrate in response to CXCL12.

To evaluate the relevance of this pathway to humans, the authors first showed that lymphomas in the brains of GFAP-LT $\alpha \beta$ mice had similar gene expression profiles to human lymphomas and responded to high-dose methotrexate, a common CNSL therapy. They also analysed biopsy samples of primary and secondary human CNSLs and found expression of several components of the NF- $\mathrm{kB}$ pathway as well as expression of CCR7 in LCs and CCL19 in brain cells.

Finally, as gliosis increases with age, and the average age of primary CNSL onset is 65 years, the authors examined whether age-related gliosis might be a risk factor for CNSL development. Older wild-type mice had evidence of gliosis and inflammatory pathway activation as compared with younger mice (11 months versus 2 months old), and larger CNSL lesions developed in these older mice when MYC-expressing LCs were injected.

The data in this study suggest that the CCL19-CCR7 signalling pathway could potentially be inhibited to prevent CNSL development. It is also plausible that this pathway might promote brain retention of other types of cancer cells (for example, during metastasis), but this would need to be investigated in other studies.

Sarah Seton-Rogers

ORIGINAL ARTICLE O'Connor, T. et al. Age-related gliosis promotes central nervous system lymphoma through CCL19-mediated tumor cell retention. Cancer Cell 36, 250-267 (2019) 